\title{
Design of Power Quality Monitoring System Based on Wireless Network
}

\author{
Ke-Li Duan \\ Alxa Power Bureau of Inner Mongolia Electric Power Group Co., Ltd. Alxa, China \\ E-mail: tdqiushipaper@163.com
}

\begin{abstract}
In order to improve the real-time power quality monitoring, this paper presents a power quality monitoring system based on ZigBee, mainly comprising three parts, these are the monitoring system of power quality, composition data communications and monitoring center. Power system operation of power quality parameters achieves a rapid and accurate remote monitoring, simple in structure, flexible expansion as well as improved automation of power quality monitoring.
\end{abstract}

Keywords-power quality; wireless internet; GPRS; monitoring system

\section{INTRODUCTION}

With the rapid development of modern science and technology, China's power electronics technology has gradually become mature. Widely used in high-power and non-linear power electronic devices caused by power quality problems have gradually emerged. Power harmonic interference, three-phase voltage imbalance, fluctuation and flicker, voltage and current disturbance and other negative phenomena after another, serious impact on power quality. Some high-precision power electronics and automation control equipment to a wide range of applications and daily life of residents in the production, which is a higher power quality requirements.

This paper presents a power quality detection scheme based on ZigBee technology to achieve the monitoring center of the detection node remotely power quality real-time, accurate detection, provide the basis for centralized management of power systems, maintenance and repair, has a certain practical value.

\section{POWER QUALITY PRINCIPLE AND DETECTION METHOD}

\section{A Basic Power Quality and Standards}

Power quality refers to the quality of electrical energy in the power system, the ideal should be symmetrical sine wave power. The main indicator of the quality of power quality voltage, current, quality, quality and quality of electricity supply. China power system nominal frequency of $50 \mathrm{~Hz}$, transformed through the transformer to a predetermined voltage level to the power supply for the user. In the idealized three-phase network, each phase voltage and current of equal amplitude, phase difference 1200. Due to a number of factors will deviate from the symmetrical sine wave, power quality problems arose. Application of such non-ideal power elements, such as electric motors, transformers, etc., lead to some of the symmetry and linearity of the load variation. Electrical component installation and improper operation may cause power quality problems [1].

Standard voltage deviation: voltage deviation is an important indicator of the basic power quality is an important criterion for judging the power supply system running deviation. When the power system during normal operation, the actual RMS voltage of a node where the percentage deviation from nominal voltage is the voltage deviation of the node. The mathematical formula is as formula (1).

$$
\delta U(\%)=\frac{U-U_{N}}{U_{N}} \times 100 \%
$$

In formula(1): $\delta U$ percentage voltage deviation; $U$ is the actual voltage $(\mathrm{KV})$; UN system nominal voltage $(\mathrm{KV})$. When the normal operation of power grids, the client allows the voltage deviation.

- The $35 \mathrm{kV}$ and above power supply voltage terminal and there are special quality requirements do not exceed the rated voltage of $\pm 10 \%$.

- $10 \mathrm{kV}$ and below the three-phase high voltage power supply and low voltage distribution the user does not exceed the rated voltage of $\pm 7 \%$.

- $220 \mathrm{~V}$ single-phase power supply voltage deviation allowable range of the rated voltage of $+7 \%$ to $10 \%$.

\section{B Grid Frequency Deviation}

Grid frequency deviation is one of the important indicators of power quality. Grid frequency has an important impact on the normal operation of power system loads, such as power plant power load on the grid frequency has strict requirements. To ensure the normal operation of electricity users must maintain the system frequency is almost constant, so that the grid frequency deviation control within a certain range is as formula(2).

$$
\delta \mathrm{f}=\mathrm{fre}-\mathrm{fN}
$$

$\delta \mathrm{f}$ is the frequency deviation; fre actual frequency; $\mathrm{fN}$ nominal frequency of the system. Frequency deviation should not exceed $\pm 0.2 \mathrm{~Hz}$, otherwise it will cause harm to systems and electrical equipment, and even a system crash.

\section{Three-Phase Voltage Unbalance}

Over the next three-phase voltage-like body should be A, B, C three-phase voltage phasor magnitude equal and uniform phase angle difference between each of 1200, such a system is called a symmetrical three-phase AC system. 
According to symmetrical components, three-phase AC power system can be decomposed into a positive sequence, negative sequence and zero sequence three symmetrical components[2]. Formula three-phase AC system voltage, current imbalance is as follows:

$$
\begin{gathered}
\varepsilon_{\mathrm{U}}=\frac{\mathrm{U}_{2}}{\mathrm{U}_{1}} \times 100 \% \\
\varepsilon_{\mathrm{I}}=\frac{\mathrm{I}_{2}}{\mathrm{I}_{1}} \times 100 \%
\end{gathered}
$$

$\varepsilon \mathrm{U}, \varepsilon \mathrm{I}$ are three-phase voltage, current imbalance; $\mathrm{U} 1$, $\mathrm{U} 2$ are the fundamental voltage positive sequence, negative sequence component rms $(\mathrm{kV})$; I1, I2, respectively fundamental current positive sequence, negative sequence component rms (kA).

Under normal circumstances, generally not more than $2 \%$, short of the three-phase voltage unbalance does not exceed $4 \%$ and must be able to ensure the normal operation of the automatic apparatus.

\section{Power Quality Detection Algorithm}

\section{1) Frequency detection}

Frequency detection is an important indicator of power quality, mature frequency measurement algorithms are two broad categories of hardware and software testing method frequency measurement frequency method. In this paper, frequency measurement method, in the design of frequency deviation detection program, it is based on the fast Fourier transform algorithm. FFT algorithm to detect frequency. FFT is a sophisticated digital filtering algorithm, when selecting the appropriate sampling frequency and data window, such algorithm can correctly model the measured signal parameters determined[3].

\section{2) Phase unbalance detection}

In this paper, three-phase voltage unbalance is detected using symmetrical components, symmetrical components is an important method for analyzing asymmetric symmetric systems running state. When the detected voltage phase imbalance, first FFT analysis of the signal to obtain a three-phase voltage fundamental amplitude and phase, then the use of symmetrical components calculated voltage positive, negative and zero sequence magnitude formula as follows:

$$
\begin{gathered}
\mathrm{U}_{1}=\frac{\mathrm{U}_{\mathrm{A}}+\alpha \mathrm{U}_{\mathrm{B}}+\alpha^{2} \mathrm{U}_{\mathrm{C}}}{3} \\
\mathrm{U}_{2}=\frac{\mathrm{U}_{\mathrm{A}}+\alpha^{2} \mathrm{U}_{\mathrm{B}}+\alpha \mathrm{U}_{\mathrm{C}}}{3} \\
\alpha=-\frac{1}{2}+\mathrm{j} \frac{3}{2}
\end{gathered}
$$

The UA, UB, UC for the three-phase voltage fundamental component.

\section{E Fast Fourier Transform (Fft) Algorithm}

The Fast Fourier Transform or simply FFT is a very effective algorithm that is applied in harmonic detection. Consider the analysis of a single frequency signal with the DC component and the harmonic signal as an example based on $\mathrm{x}(\mathrm{t})$, the discrete time signal is obtained from the sampling frequency $f_{s}$, as shown in formula (8).

$$
x(n)=A_{0}+\cos \left(2 \pi \frac{f_{i}}{f_{s}} n+\varphi_{i}\right)
$$

Where $A_{0}$ is the amplitude of the DC component, $A_{i}, f_{i}$, $\varphi_{\mathrm{i}}$ are amplitude of $\mathrm{AC}$ component, frequency and initial phase respectively; $\mathrm{n}=0,1, \cdots, \mathrm{N}-1, \mathrm{~N}$ is number of sampling points. The FFT for the signal is as formula (9).

$$
X(k)=A_{0}+\frac{A_{i}}{2}\left(e^{j \varphi_{1}}+e^{-j \varphi_{1}}\right)
$$

\section{WHOLE SYSTEM DESIGN}

Power quality monitoring system designed in this paper based on wireless network mainly includes three parts, power quality detection node, ZigBee receiving, sending and monitoring center. Construction of WSNs by local detection node ZigBee technology, power quality data collected after the detection node spread ZigBee module, and then transmits the ZigBee module to the local terminal, and finally sent by GPRS to a remote monitoring center. This system can achieve real-time detection of power quality data, power quality data transfer and terminal data monitoring and analysis capabilities.

Power quality detection node function is responsible for collecting power quality-related data, such as voltage, current, and power frequency and so on. According to the characteristics of power system equipment, the settings on each cycle of the sampling 128 points, you can do real-time data collection. Power quality detection system structure shown in Figure 1 node, is mainly composed of voltage transformers, current transformers, circuit information and ZigBee conditioning circuit module. Voltage and current sensing transformer three-phase grid voltage and current signals. After the signal conditioning circuit amplifies, filters, use CC2530 comes with an A / D conversion is completed analog to digital conversion. By ZigBee module sends data to a local monitoring terminal. The power system configuration diagram of detection node is as Fig.1.

\begin{tabular}{|c|c|c|}
\hline \multirow{2}{*}{ PT,CT } & $\begin{array}{c}\text { Signal Processing } \\
\text { Circuit }\end{array}$ \\
\cline { 3 - 3 } & & ZigBee Module \\
CC2530
\end{tabular}

Figure 1. Power system configuration diagram of detection node.

The local monitoring terminal system structure is as in Fig.2, after the power quality data collection is completed, the local display module LCD and SD card for data display and storage. Responsible for receiving and sending data to the local terminal ZigBee module. The automatic networking capabilities of ZigBee, fully achieve real-time data transmission. The communication system of each detection node local terminal and a remote monitoring center through GPRS network to achieve. GPRS network communication is the basis of the TCP / IP protocol, access to the GPRS network can directly access the Internet 
network. The system uses to achieve TCP / IP protocol directly on the PC, so that it is directly connected with GPRS communication module back into the GPRS network to communicate with a remote monitoring center. Remote monitoring terminal can communicate simultaneously with multiple WSNs network to obtain the grid power quality conditions in different regions [4].

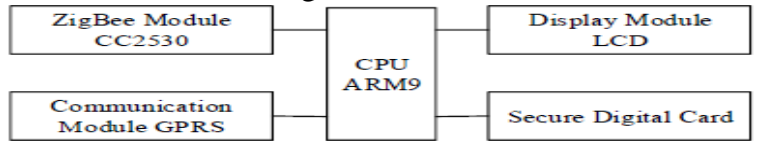

Figure 2. Local monitoring terminal system configuration diagram.

Remote monitoring terminal area energy data monitoring and analysis. After the data collected to reach the remote terminal, which can be saved and display of power quality data. Standard power quality parameters were compared, real-time monitoring of operational quality electricity.

\section{SOFTWARE DESIGN OF SYSTEM}

Local monitoring terminal responsible for setting up a wireless network, Fourier transform, to obtain operating parameters of power quality and power quality data transmission to the remote terminal via GPRS, the flowchart of monitoring system is as Figure 3.

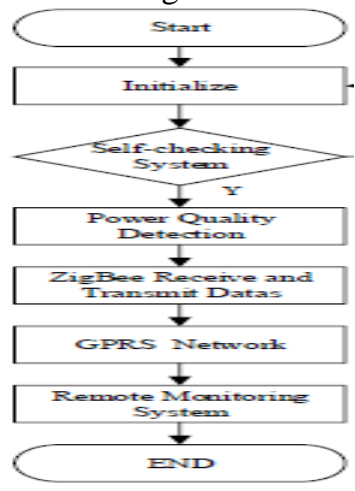

Figure 3. Flowchart of monitoring system.
After the system is powered on, power quality monitoring modules and ZigBee module begins to initialize[5]. First ZigBee module to check the communication and power quality testing equipment and routers. ZigBee module sends data via GPRS, the data is transmitted, the data is automatically transmitted to the terminal device. Terminal equipment for data monitoring and analysis functions, and displayed and stored in the terminal.

\section{CONCLUSIONS}

This article gave a detailed description of the design of power quality monitoring system based on wireless sensor networks. The system employs wireless sensor networks to measure real-time data of power quality data acquisition, and through the GPRS network transmits data to the remote monitoring terminal. Monitoring personnel can observe the power system operation status, and power quality data needed for statistical analysis, thus ensuring decision making based on coordinated control. The system realizes automated real-time monitoring of power quality, has a structured system architecture, scalability, and has some promotional and application value.

\section{REFERENCES}

[1] Zhao Suhai, Power Quality Detection System Based on Wireless Sensor Network Research[D].Anhui: Anhui University of Science and Technology,2012

[2] $\mathrm{Li} \mathrm{Ji}$-xin.Virtual Instrument Technology and Development Trend[J] .ElectronicMaterial\&Electronic Technology 2008,32(3): $39-46$.

[3] Liu Hui,Application Research of Power Quality Monitoring System Based on wireless Channel.Shan Dong,ShanDong University,2011.

[4] ZHANG Bin,SUN Jing. A Power Quality Analysis Method Based on Mallat Algorithmand Fast Fourier Transform[J].Power System Technology 2007 31(19): 35-40.

[5] Yang Huanghua, Research and Design of Power Quality Monitoring System[D],Shan Xi, TaiyuanUniversity of Science and Technology,2011. 